\title{
Seismic Response of Double Metro Tunnels Built in Deep Soft-Soil
}

\author{
Guy Ange N'goran Ehouman ${ }^{1 *}$ Zhang Jing-Li ${ }^{1}$
}

1. School of Civil Engineering an Architecture, Zhejiang University of Science and Technology, No.318, Liu-he Road, Hangzhou, Zhejiang Province, 310023, China

2. School of Civil Engineering an Architecture, Zhejiang University of Science and Technology, No.318, Liu-he Road, Hangzhou, Zhejiang Province, 310023, China

* E-mail of the corresponding author: guyangengoran@gmail.com

\begin{abstract}
Rapid development and urbanization have led to the use of underground structures along the world for a wide range of applications including tunnel for subways, railways, highways, material storage, sewage and water transport. The safety of these facilities during seismic events such as recent earthquake in Japan, Taiwan and Turkey becomes more and more problematic due to the complex soil-structure interaction (SSI). The major factor causing severe damages to metro structures is the large deformations imposed by surrounding soils. Thus, for a subway tunnel structure embedded in the soft soil, the study of nonlinear dynamic response of soil-structure interaction during earthquake is important. Nowadays, numerous tools of Finite Element (FE) analysis has been developed to simulate and analyse the tunnel behaviour in order to mitigate its damages during seismic events. In this paper, the displacement response of double metro tunnels under two different earthquake waves is investigate using a Finite Element program (ABAQUS) in 2D plane strain condition, by means of nonlinear dynamic behaviour of soil and tunnels in three different working conditions by varying the embedded depth of the tunnels in soil. The results show that the displacement response of double tunnels built in soft soil is related to the characteristics of the input ground motion, the nature of surrounding soil and the buried depth of the tunnel structures, the peak ground acceleration (PGA) and the SSI effect has an important influence on the displacement response of tunnel structures.
\end{abstract}

Keywords: double metro tunnels, soft soil, nonlinear, dynamic analysis, ground motion, displacement response, SSI, FE, PGA, ABAQUS

DOI: $10.7176 /$ ISDE/12-2-04

Publication date: June $30^{\text {th }} 2021$

\section{Introduction}

The development and utilization of underground space becomes a new era hot issue for civil engineering in the 21 st century. Among them, the subway is one of the mean vehicles of people's daily travel, and the seismic performance requirements for its structure is particularly high ${ }^{[1]}$. In the past, seismic design of underground structures have received considerably less attention than that of surface structures because of two reason. Firstly, because in the early stage of underground engineering construction, it was considered that the underground structures were buried in the rock and soil layer their influence on earthquake was very small and also, the surrounding rock and soil layer constitute a natural protective layer. Secondly, because at that time, there is significantly less information available for the design of underground structures ${ }^{[2-3-4]}$. But in recent large earthquakes event including the 1995 Kobe, Japan earthquake, the 1999 Chi-Chi, Taiwan earthquake and the 1999 Kocaeli, Turkey earthquake in which subway stations, tunnels and utility tunnels have experienced a significant number of damage shaken underground engineering and brought a great impact to this traditional concept, which changed that perception of earthquake workers ${ }^{[5]}$. Several researches has been carry out along the world including Dowding C. R., 1978, Owen, 1981, GN, Sharma, 1991, Wang W. L., 2001 and Wang ZZ, 2009 and more than 192 case studies ${ }^{[6-7-8-9]}$ were considered and studied with the following objectives (1) attenuation relationship (2) magnitude and epicenter distance and (3) Peak ground acceleration. And these different works yielded the following conclusion:

- The ovaling effect on circular tunnels and racking effect on rectangular tunnels are found to be highly dependent on the relative stiffness between the tunnel lining and the surrounding ground, which makes the soilstructure interaction one of the most important factors in the seismic design and evaluation for tunnel structures.

-Tunnels built in rock medium responded with a little damage with a peak acceleration below $0.4 \mathrm{~g}$, 
- Cut and cover tunnels and shallow tunnels tend to be more vulnerable,

-Deeper tunnels seems to be safer and damages are increased with the earthquake magnitude and decrease epicenter,

-Damages to tunnel are less with PGAs less than $0.2 \mathrm{~g}$ whiles damages with PGAs between $0.2 \mathrm{~g}-0.5 \mathrm{~g}$ incurred slightly too heavy damages.

This present research work will focus specially on the displacement response of double metro tunnels system under earthquake loading. To do so, a 2D Finite Element Module will be developed in plane strain condition using AABAQUS Software to model the nonlinear dynamic behaviour of soil and double tunnels. The analysis will be conducted in three different working conditions by varying the depth of the tunnel structures. At the end of simulations, the results will be compared and studied.

\section{Outline of the Problem}

The Nanjing line 1 runs mainly in a north-south direction. The line starts at Magaoqiao station in the north, heading southwest to CPU (China Pharmaceutical University). The construction of the line 1 began in the year 2000 and was inaugurated on September 3, 2005, with sixteen stations and a length of 21.72 kilometers (13.50 mi). $14.33 \mathrm{~km}$ of the line track runs underground while $7.39 \mathrm{~km}$ of the track run on or above ground. In fact, the structure is built in a thick soft soil ${ }^{[10]}$ and the depth of line 1 is from $9 \mathrm{~m}-14 \mathrm{~m}$ when the metro line is transferred from underground to the ground and the thickness of the over bedding soil layer is small. For this reason, this study will consider three working conditions $(3 \mathrm{~m}, 9 \mathrm{~m}$, and $14 \mathrm{~m})$. The tunnel lining has a thickness of $0.7 \mathrm{~m}$, the external and internal diameters are respectively $5.5 \mathrm{~m}$ and $6.2 \mathrm{~m}$. The thickness of the duct pieces is $35 \mathrm{~cm}$, and the metro concrete is C50. The shaking damages of earthquake event on a metro line are characterized by the overburden depth and the properties of the surrounding soil. The larger deformation of the surrounding soil cause severe damages to metro tunnel because the inertia of the surrounding soil is larger relative to the structure and in an event of seismic loading the soil-metro structure interaction cannot be neglected.

\section{Modelling and the Parameters Selected}

\subsection{Finite element model of subway tunnel and soil}

In this study, a 2-D finite element model will be developed in plain strain by using ABAQUS software. The tunnels lining are chosen as a reinforce concrete with a circular shape and the ground is modelled with ten horizontal soil layer profiles. The soil dimensions are $\left(100^{*} 61\right)$, respectively as horizontal and vertical distances. The tunnels are assumed to be constructed in three different overburden depth, $3 \mathrm{~m}, 9 \mathrm{~m}$, and $14 \mathrm{~m}$, which leads to three working conditions. The vertical distance between the two tunnels is $13 \mathrm{~m}$ and the numerical analysis of the soil and the metro tunnel is modelled using ABAQUS user subroutine to define the material properties to account for the nonlinear dynamic behaviour of the soil and the metro tunnel during earthquake loading. The module is meshed with CPE4R element type with quadrilateral element shape and a pressure of 20kpa is applied uniformly at the top of the ground surface. Two different earthquake waves are applied in the horizontal direction at the bedrock of the soil profile with three different peak accelerations. Then, the dynamic implicit analysis is conducted with ABAQUS software to simulate the model. Fig.1, and Fig.2 show the finite element mesh of the soil and tunnel lining.

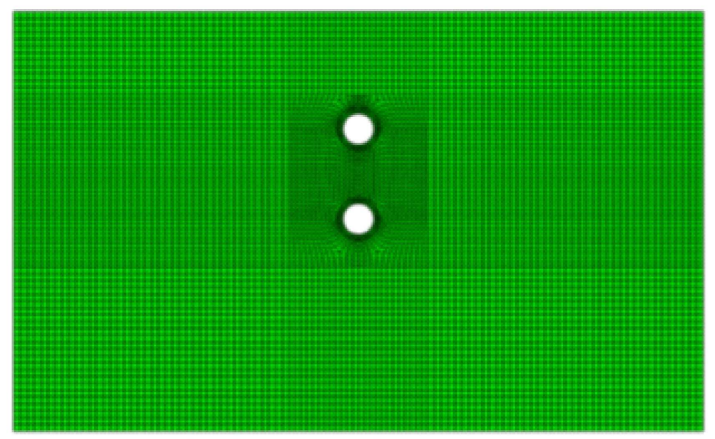

Figure1. Finite element mesh of soil

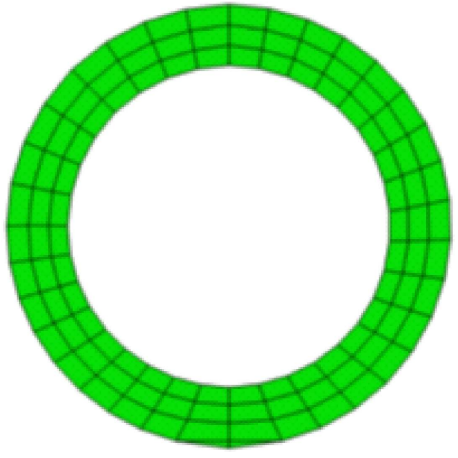

Figure2. Finite element mesh of tunnel 
Table1. Concrete Tensile Behaviour

\begin{tabular}{|c|c|}
\hline Yield stress $(\mathrm{kN} / \mathrm{m} 2)$ & Displacement $(\mathrm{mm})$ \\
\hline 2900 & 0 \\
\hline 2020 & $5.52 \mathrm{E}-05$ \\
\hline 1410 & $1.03 \mathrm{E}-05$ \\
\hline 979 & $1.45 \mathrm{E}-04$ \\
\hline 682 & $1.83 \mathrm{E}-04$ \\
\hline 475 & $2.19 \mathrm{E}-04$ \\
\hline 330 & $2.53 \mathrm{E}-04$ \\
\hline 230 & $2.86 \mathrm{E}-04$ \\
\hline 160 & $3.18 \mathrm{E}-04$ \\
\hline 112 & $3.50 \mathrm{E}-04$ \\
\hline 77.6 & $3.81 \mathrm{E}-04$ \\
\hline 54.1 & $4.11 \mathrm{E}-04$ \\
\hline 37.6 & $4.42 \mathrm{E}-04$ \\
\hline
\end{tabular}

Table2. Concrete Compressive Behaviour

\begin{tabular}{|l|l|}
\hline Yield stress $(\mathrm{kN} / \mathrm{m} 2)$ & Compressive strain \\
\hline 4690 & 0 \\
\hline 6830 & 0.001 \\
\hline
\end{tabular}

Table3. Soil Constitutive Parameters

\begin{tabular}{|l|c|c|c|c|c|c|c|}
\hline Soil $\mathrm{n}_{0}$ & Thickness & $\begin{array}{c}\text { Soil } \\
\text { description }\end{array}$ & $\begin{array}{c}\text { Gravity } \\
\text { KN/m3 }\end{array}$ & $\begin{array}{c}\text { Young Modulus } \\
(\mathrm{MPa})\end{array}$ & $\begin{array}{c}\text { Poisson } \\
\text { Ratio }\end{array}$ & $\begin{array}{c}\text { Shear wave Velocity } \\
(\mathrm{m} / \mathrm{s})\end{array}$ & $\begin{array}{c}\text { Friction } \\
\text { angle } \Phi\end{array}$ \\
\hline 1 & 2.0 & Mucky soil & 19.0 & 1.0 & 0.45 & 114 & 16 \\
\hline 2 & 2.0 & Mucky silty & 17.8 & 1.0 & 0.45 & 129.1 & 16 \\
\hline 3 & 4.0 & Silt and sand & 19.0 & 5.2 & 0.35 & 152.7 & 26 \\
\hline 4 & 3.0 & Mealy sand & 20.5 & 7.5 & 0.30 & 137.1 & 30 \\
\hline 5 & 3.0 & Mucky soil & 19.3 & 2.1 & 0.45 & 128.5 & 16 \\
\hline 6 & 9.0 & Fine sand & 18.9 & 10.0 & 0.30 & 172.7 & 27 \\
\hline 7 & 12.5 & Silt-fine sand & 21.2 & 11.1 & 0.32 & 205.8 & 30 \\
\hline 8 & 10.3 & Fine sand & 18.9 & 11.7 & 0.30 & 236.3 & 27 \\
\hline 9 & 5.2 & Mealy sand & 20.5 & 12.0 & 0.32 & 263.2 & 30 \\
\hline 10 & 10.0 & Clay & 19.3 & 3.2 & 0.42 & 491.6 & 21 \\
\hline
\end{tabular}

\section{Boundary Condition}

The boundary conditions as adopted for double tunnels analysis in this study is made so that, the wave refraction and reflection of the vertical model boundaries can be avoided. According to Meissner (1996), the choice of sufficient mesh dimension is made so that, the stresses at the model boundaries should not be influenced by the tunnel excavation, and therefore, recommends the use of (4-5) times the tunnel diameter from the tunnel center 
line to the vertical mesh boundaries and (2-3) times from the tunnel center point to the bottom boundary. For the vertical boundaries, the horizontal displacement/rotation is restrain and the vertical displacement/rotation is left free. The bottom boundary has total fixities restraining both horizontal and vertical displacements/rotation. The upper horizontal boundary has no fixities and is left free to displace.

\section{Earthquake Wave Selection}

In this present study, two-time histories earthquake acceleration wave are used for the analysis. The first one is the El Centro acceleration recorded during the 1940, Imperial Valley Earthquake ${ }^{[1]}$ and the second one is the Taft acceleration recorded during the 1952, Kern County, California Earthquake ${ }^{[12]}$. Fig 3.7shows the unscaled acceleration time history of the two selected waves (El-Centro and the Taft input wave). The input acceleration is scaled into three peaks ground accelerations $0.053 \mathrm{~g}, 0.116 \mathrm{~g}$ and $0.154 \mathrm{~g}$ to simulate the probability of exceedance $63 \%, 10 \%, 3 \%$ in 100 years of the site.
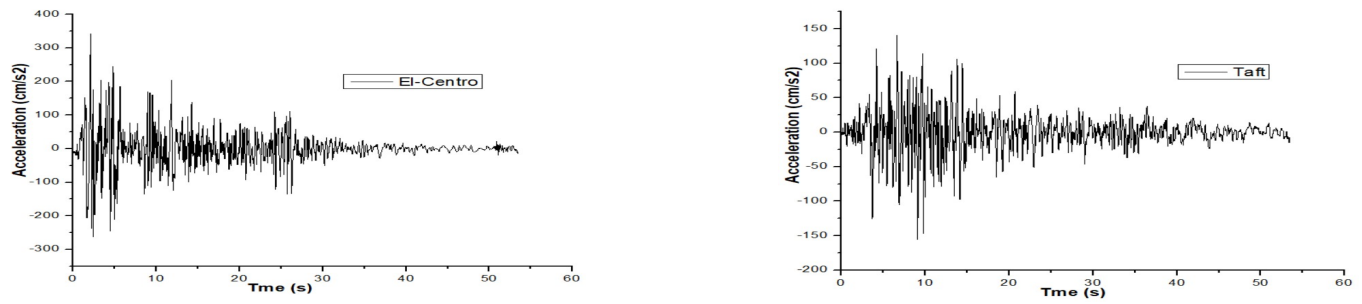

Figure3. El-Centro and Taft wave unscaled acceleration time histories.

\section{Result and Analysis of horizontal displacement}
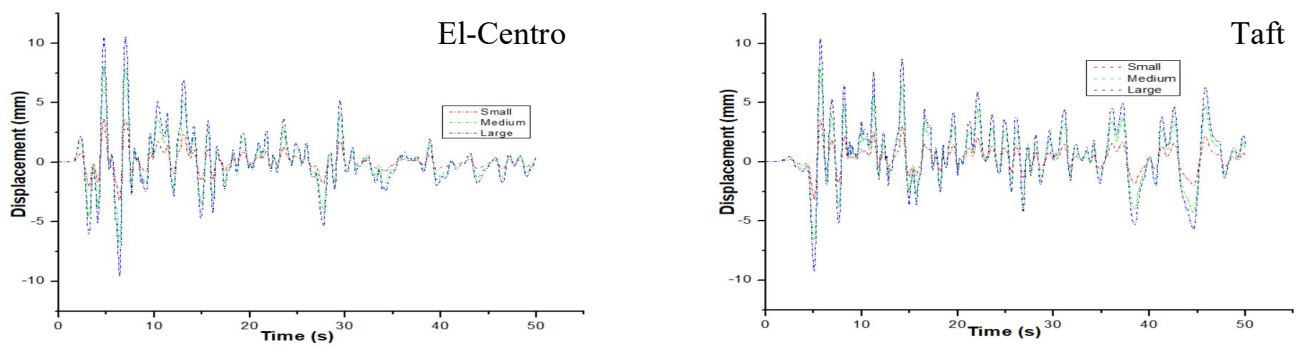

Figure4. Horizontal relative displacement time histories between the top and bottom of the upper tunnel in first working condition with El-Centro and Taft input.
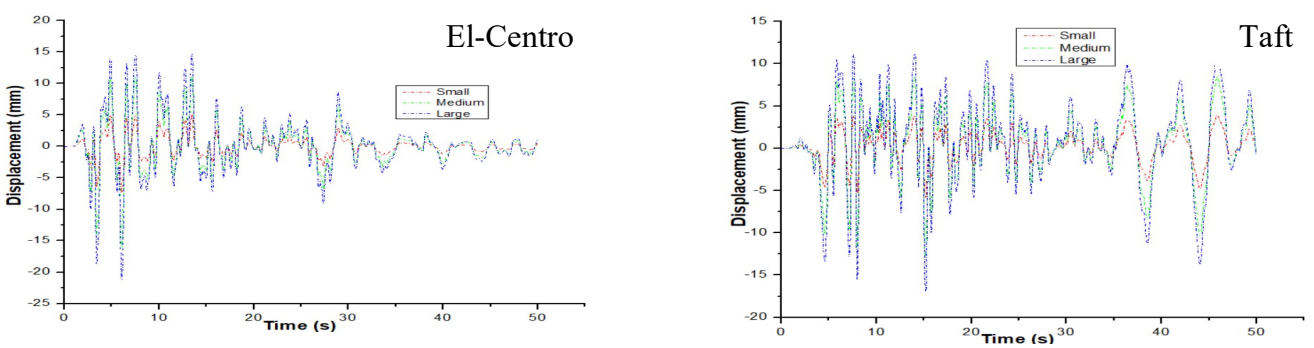

Figure5. Horizontal relative displacement time histories between the top and bottom of the lower tunnel in first working condition with El-Centro and Taft input. 

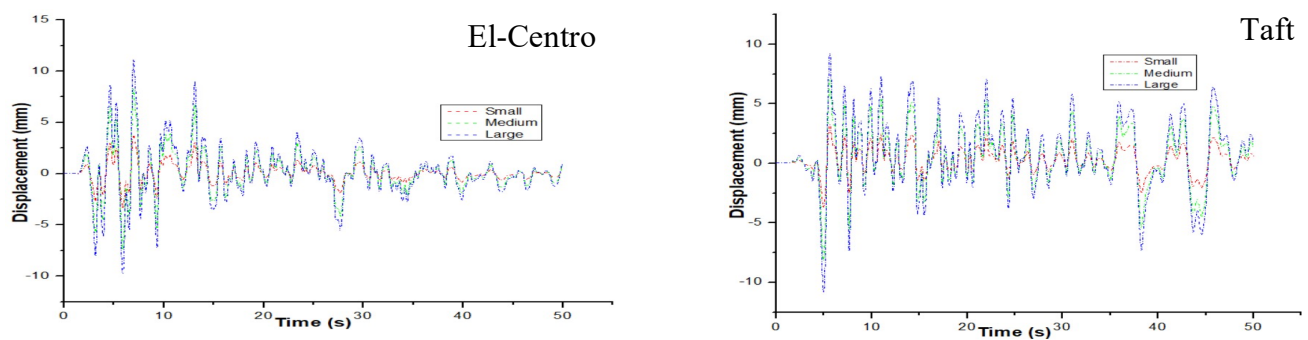

Figure6. Horizontal relative displacement time histories between the top and bottom of the upper tunnel in the second working condition with El-Centro and Taft input.
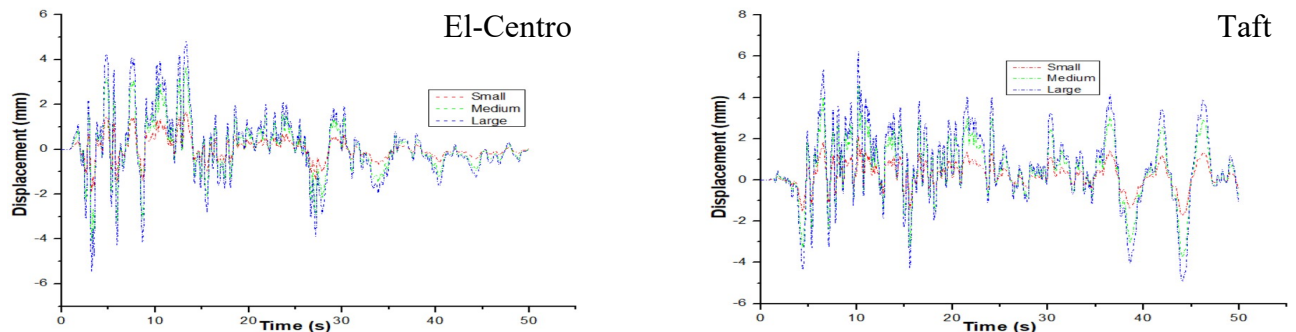

Figure7. Horizontal relative displacement time histories between the top and bottom of the lower tunnel in the second working condition with El-Centro and Taft input.
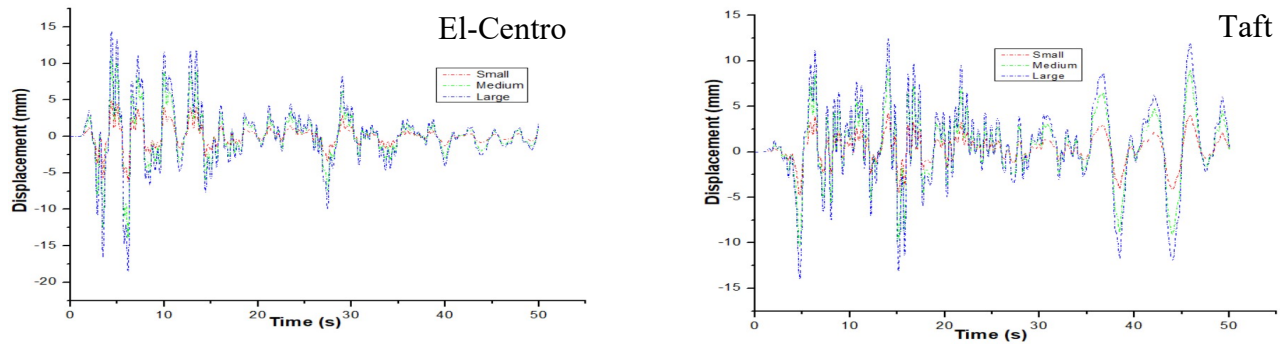

Figure8. Horizontal relative displacement time histories between the top and bottom of the upper tunnel in third working condition with El-Centro and Taft input. 

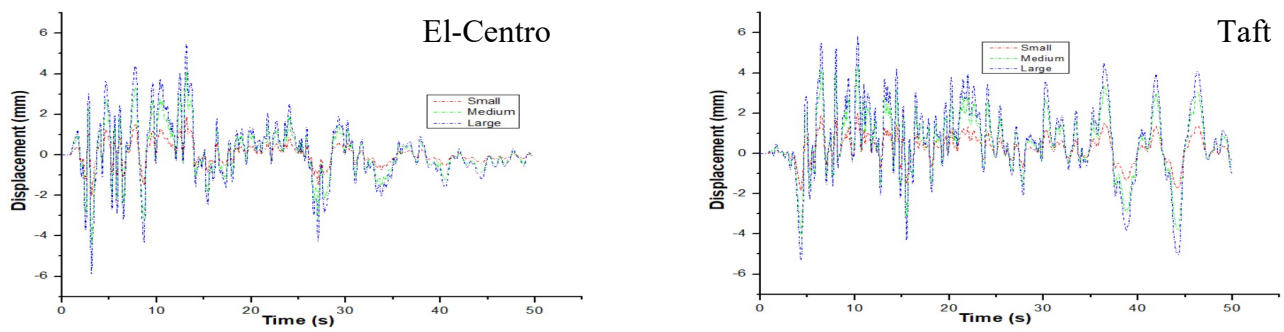

Figure.9 Horizontal relative displacement time histories between the top and bottom of the lower tunnel in third working condition with El-Centro and Taft input.

Fig.4 to 9 show the horizontal relative displacement of double tunnels in three different working conditions with two different input waves (El-Centro and Taft waves) scaled at $0.053 \mathrm{~g}, 0.116 \mathrm{~g}$ and $0.154 \mathrm{~g}$. The first working condition is $3 \mathrm{~m}$ depth, the second is $9 \mathrm{~m}$ depth and the third is $14 \mathrm{~m}$ depth. The figures show an increase in relative displacement with the lower tunnel then the upper one under the two input waves in the first working condition. The relative displacement in this condition is large with El-Centro input. In the second working condition, figures show an increase in relative displacement with the upper tunnel then a decrease with the lower one under the two input waves. In the third working condition, figures show an increase in relative displacement with the upper tunnel then a decrease with the lower one under the two input waves.

Table5. Maximum relative displacement under different working condition

\begin{tabular}{|c|c|c|c|c|c|c|c|c|}
\hline \multirow{2}{*}{\multicolumn{3}{|c|}{ Input Wave }} & \multicolumn{2}{|c|}{ 3m Depth } & \multicolumn{2}{|c|}{ 9m Depth } & \multicolumn{2}{|c|}{$14 \mathrm{~m}$ Depth } \\
\hline & & & $\Delta S(\mathrm{~mm})$ & $\zeta$ & $\Delta S(\mathrm{~mm})$ & $\zeta$ & $\Delta S(\mathrm{~mm})$ & $\zeta$ \\
\hline \multirow{6}{*}{$\begin{array}{l}\text { Upper } \\
\text { Tunnel }\end{array}$} & \multirow{2}{*}{ Small } & El-Centro & 3.694 & \multirow{2}{*}{1.019} & 3.820 & \multirow{2}{*}{1.029} & 6.381 & \multirow{2}{*}{1.326} \\
\hline & & Taft & 3.627 & & 3.711 & & 4.814 & \\
\hline & \multirow{2}{*}{ Medium } & El-Centro & 8.016 & \multirow{2}{*}{1.015} & 8.331 & \multirow{2}{*}{1.021} & 13.947 & \multirow{2}{*}{1.324} \\
\hline & & Taft & 7.894 & & 8.157 & & 10.534 & \\
\hline & \multirow{2}{*}{ Large } & El-Centro & 10.613 & \multirow{2}{*}{1.018} & 11.110 & \multirow{2}{*}{1.023} & 18.467 & \multirow{2}{*}{1.318} \\
\hline & & Taft & 10.425 & & 10.865 & & 14.008 & \\
\hline \multirow{6}{*}{$\begin{array}{l}\text { Lower } \\
\text { Tunnel }\end{array}$} & \multirow{2}{*}{ Small } & El-Centro & 7.375 & \multirow{2}{*}{1.258} & 1.876 & \multirow{2}{*}{0.884} & 2.034 & \multirow{2}{*}{1.014} \\
\hline & & Taft & 5.862 & & 2.122 & & 2.005 & \\
\hline & \multirow{2}{*}{ Medium } & El-Centro & 16.111 & \multirow{2}{*}{1.258} & 4.097 & \multirow{2}{*}{0.879} & 4.451 & \multirow{2}{*}{1.013} \\
\hline & & Taft & 12.803 & & 4.659 & & 4.394 & \\
\hline & \multirow{2}{*}{ Large } & El-Centro & 21.345 & \multirow{2}{*}{1.257} & 5.437 & \multirow{2}{*}{0.876} & 5.910 & \multirow{2}{*}{1.011} \\
\hline & & Taft & 16.979 & & 6.207 & & 5.844 & \\
\hline
\end{tabular}

Table6. Depth influence coefficient El-Centro wave

\begin{tabular}{|c|c|c|c|c|c|c|c|}
\hline \multirow{3}{*}{\multicolumn{2}{|c|}{ Depth }} & \multicolumn{6}{|c|}{ El-Centro Input } \\
\hline & & \multicolumn{2}{|c|}{ Small } & \multicolumn{2}{|c|}{ Medium } & \multicolumn{2}{|c|}{ Large } \\
\hline & & $\Delta s(\mathrm{~mm})$ & $\xi$ & $\Delta s(\mathrm{~mm})$ & $\xi$ & $\Delta s(\mathrm{~mm})$ & $\xi$ \\
\hline \multirow{3}{*}{$\begin{array}{l}\text { Upper } \\
\text { Tunnel }\end{array}$} & $3 \mathrm{~m}$ & 3.694 & 5 & 8.016 & $=$ & 10.613 & $\pi$ \\
\hline & $9 m$ & 3.820 & 0.967 & 8.331 & 0.962 & 11.110 & 0.955 \\
\hline & $14 \mathrm{~m}$ & 6.381 & 0.599 & 13.947 & 0.597 & 18.467 & 0.602 \\
\hline \multirow{4}{*}{$\begin{array}{l}\text { Lower } \\
\text { Tunnel }\end{array}$} & $>$ & $\Delta s(\mathrm{~cm})$ & $\zeta$ & $\Delta s(\mathrm{~cm})$ & $\zeta$ & $\Delta s(\mathrm{~cm})$ & $\zeta$ \\
\hline & $3 \mathrm{~m}$ & 7.375 & 1 & 16.111 & & 21.345 & \\
\hline & $9 \mathrm{~m}$ & 1.876 & 3.931 & 4.097 & 3.932 & 5.437 & 3.926 \\
\hline & $14 \mathrm{~m}$ & 2.034 & 0.922 & 4.451 & 0.920 & 5.910 & 0.920 \\
\hline
\end{tabular}


Table7. Depth influence coefficient Taft waves

\begin{tabular}{|c|c|c|c|c|c|c|c|}
\hline \multirow{3}{*}{\multicolumn{2}{|c|}{ Depth }} & \multicolumn{6}{|c|}{ Taft Input } \\
\hline & & \multicolumn{2}{|c|}{ Small } & \multicolumn{2}{|c|}{ Medium } & \multicolumn{2}{|c|}{ Large } \\
\hline & & $\Delta s(\mathrm{~mm})$ & $\xi$ & $\Delta s(\mathrm{~mm})$ & $\xi$ & $\Delta s(\mathrm{~mm})$ & $\xi$ \\
\hline \multirow{3}{*}{$\begin{array}{l}\text { Upper } \\
\text { Tunnel }\end{array}$} & $3 \mathrm{~m}$ & 3.627 & & 7.894 & 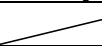 & 10.425 & \\
\hline & $9 m$ & 3.711 & 0.997 & 8.157 & 0.968 & 10.865 & 0.960 \\
\hline & $14 \mathrm{~m}$ & 4.814 & 0.771 & 10.534 & 0.774 & 14.008 & 0.776 \\
\hline \multirow{4}{*}{$\begin{array}{l}\text { Lower } \\
\text { Tunnel }\end{array}$} & $><$ & $\Delta s(\mathrm{~mm})$ & $\zeta$ & $\Delta s(\mathrm{~mm})$ & $\zeta$ & $\Delta s(\mathrm{~mm})$ & $\zeta$ \\
\hline & $3 \mathrm{~m}$ & 5.862 & & 12.803 & & 16.979 & \\
\hline & $9 \mathrm{~m}$ & 2.122 & 2.762 & 4.659 & 2.748 & 6.207 & 2.735 \\
\hline & $14 \mathrm{~m}$ & 2.005 & 1.058 & 4.394 & 1.060 & 5.844 & 1.062 \\
\hline
\end{tabular}

Table5, 6 and 7 show the maximum horizontal relative displacement of upper and lower tunnels under different working condition and the depth influence coefficient of El-Centro and Taft waves respectively. The depth influence coefficient is defined as the horizontal relative displacement when embedding depth is $3 \mathrm{~m}$ over that of $9 \mathrm{~m}$ embedding depth and when the embedding depth is $9 \mathrm{~m}$ over that of $14 \mathrm{~m}$ embedding depth.

From horizontal displacement time history curves, the comparison analysis of maximum relative displacement and depth influence coefficient, we can make the following interpretations:

* By comparing the horizontal relative displacement under two different earthquake waves (El-Centro and Taft), the results show that the relative displacement of the metro tunnel with El-Centro input is larger than the relative displacement in Taft case. This indicates that the earthquake response of metro tunnel is related to the characteristics of the input ground motion.

*By Comparing the relative displacement between the top and bottom of the tunnels under the three working conditions, $(3 \mathrm{~m}, 9 \mathrm{~m}$, and $14 \mathrm{~m})$, it appears that the horizontal relative displacement is maximum in the first working condition when the embedding depth is $3 \mathrm{~m}$. Also, from comparative results of the relative displacement between the top and bottom of the upper and lower tunnels, it can be seen that the upper tunnel displays the largest relative displacement except in the first working condition ( $3 \mathrm{~m}$ depth). These result indicate that the buried depth of tunnel structure and the nature of soil around have a great influence on tunnel displacement response.

* For a specific working condition with the same embedding depth, the increase of the peak ground acceleration leads to an increasing of the horizontal relative displacement between the top and bottom of the tunnel structure. The maximum relative displacement appears between $5 \sim 7 \mathrm{~s}$ when inputting El-Centro waves, and between 14 16s when inputting Taft waves. When the depth is small, the depth influence coefficient $\xi$ is large. Indeed, these results mean that the displacement response of tunnel structure is sensitive to the peak ground acceleration and the SSI effect has an important influence on tunnel response.

\section{Summary}

In this paper, the horizontal displacement response of double tunnel structures built in thick soft soil at different embedded depth and subjected to two different earthquake waves is investigated. Based on the results of the displacement curves, the following conclusions can be established: (1) the seismic response of metro tunnels built in soft soil is related to the characteristics of the input ground motion. (2) The nature of surrounding soil and the buried depth of the tunnel structure have a great influence on the displacement response metro tunnel; increase the buried depth help to improve the safety of tunnel structure. (3) The peak ground acceleration and the SSI effect have an important influence on the displacement response of tunnel structure.

As future works perspective, we plan to:

(1) Develop a single model of metro tunnel with the same conditions as the double tunnels, and both results will be compared and studied.

(2) Develop a model by varying the poisons ration and the friction angle to investigate their influence on such structures when subjected earthquake loading. 
(3) Develop a model by adopting different diameters in order to investigate on the role that plays the thickness of tunnel lining during seismic shaking.

\section{References}

[1] Sakyi, K.S., Benjamin, K., Godson, k. (2018), "Seismic Response Analysis of Underground Structures"J. Enviro. Earth Sci 8 (5), 48.

[2] Zhixin Z and Jiren X 2003 J. In 1995 the Japanese earthquake in Kobe building collapse and the edge of the $\mathrm{S}$ wave and surface wave interference on the secondary Basin. Chinese Science Bulletin. 482566.

[3] Wang, J.N., 1993. Seismic Design of Tunnels, A State-of-the-Art Approach. Monograph 7, Parsons Brinkerhoff Quade and Douglas, Inc., New York.

[4] Youssef MA Hashash, Jeffrey J Hook, Birger Schmidt, I John, Chiang Yao. Seismic design and analysis of underground structures, (Tunneling and underground space technology). p248.

[5] Longjun X and Lili X 2005 J. Some considerations on the physical measure of seismic intensity. Acta Seismologica Sinica. 27656.

[6] Dowding, C.H., Rozen, A., 1978. Damage to rock tunnels from earthquake shaking. J. Geotech. Eng. Div., ASCE 104, (2), 175-191.

[7] Sharma S, Judd WR (1991) Underground opening damage from earthquakes. Engineering Geology. 30 (3-4), 263-276

[8] Wang WL, Wang TT, Su JJ, Lin CH, Seng CR, Huang TH (2001), Assessment of damage in mountain tunnels due to the Taiwan Chi-Chi earthquake. Tunnel Underground Space Technology16, (3), 133-150.

[9] Wang, J.N., and Munfakh, G.A. (2001), "Seismic Design of Tunnels", WIT Transaction on the Built Environment 57.

[10]WIKIPEDIA. Retrieved from https://en.wikipedia.org/wiki/Line_1,_Nanjing_Metro

[11] Ground-Motion Virtual Data Center (VDC), El-Centro Earthquake Wave. Retrieved from https://strongmotioncenter.org/vdc/waveform_data/smdb/1940/c/139u37el.c0a

[12] Ground-Motion Virtual Data Center (VDC), Taft Earthquake Wave. Retrieved from https://strongmotioncenter.org/vdc/waveform_data/smdb/1952/c/203e53ta.f0a 